\title{
Gold Nanorods Coated with Multilayer Polyelectrolyte as Intracellular delivery Vector of Antisense Oligonucleotides
}

\author{
Shouhui Chen, Yuxuan Ji, Qiong Lian, Yanli Wen, Hebai Shen, Nengqin Jia \\ Department of Chemistry, College of Life and Environmental Science, Shanghai Normal University, Shanghai 200234, China \\ *Corresponding author. Email address: nqjia@ shnu.edu.cn
}

\begin{abstract}
A novel functionaliztion of gold nanorods (GNRs) intracellular delivery system was prepared via electrostatically layer-by-layer assemblying, in which multilayer polyelectrolyte-coated GNRs were used as vector and antisense oligodeoxynucleotides (ASODNs) as therapeutic gene. The as-prepared positively charged GNRs were firstly modified with two successive polyelectrolyte layers of the negatively charged poly(sodium-4-styrenesulfonate) (PSS) and positively charged polyenthylenimine (PEI) in order to greatly improve the biocompatibility of these cetyltrimethylammonium bromide(CTAB)-coated GNRs and facilitate further biofunctionalization. The multilayered polyelectrolyte functionalized GNRs were characterized by UV-vis spectroscopy, transmission electron microscopy (TEM) and MTT assay. The polyelectrolytes-coated GNRs were then used as vector for ASODNs. Electrochemical impedance spectroscopy(EIS) was employed to confirm the formation of the GNRs-ASODNs conjugates through electrostatic interaction. Furthermore, cellular uptake and delivery efficiency of the GNRs-ASODNs conjugates as well as cellular apoptosis induced by the ASODNs transfected with gold nanorods were investigated by confocal microscopy and flow cytometry, exhibiting efficient intracellular delivery and improved antitumor activity of ASODNs by the polyelectrolytes-coated GNRs carriers.
\end{abstract}

Keywords: Gold nanorods; Polyelectrolytes; Antisense oligodeoxynucleotides; Biocompatibility; Intracellular delivery vector

Citation: S. Chen, et al. Gold nanorods coated with multilayer polyelectrolyte as intracellular delivery vector of antisense oligonucleotides. Nano Biomed Eng 2010 ; 2(1): 15-23. DOI: 10.5101/nbe.v2i1.p15-23

\section{Introduction}

Antisense technology has emerged as an exciting and promising strategy of cancer therapy $[1,2]$. The principle of this technology is the sequence-specific binding of an antisense oligonucleotide to target mRNA, thereby leading to the inhibition of specific gene expression and consequently to tumor cells apoptosis.

Telomerase is a unique ribonucleoprotein enzyme that is responsible for adding the telomeric repeats onto the 3'ends of chromosomes [3]. Telomerase is considered as a promising anticancer target not only for cancer diagnosis but also for the development of novel therapeutic agents, because it is active in majority of malignant tumors but not in most normal somatic cells $[4,5]$. Antisense oligonucleotides (ASODNs) as novel anticancer agents are an area of heightened interest in the field of telomerase inhibition [6]. However, practical applications of ASODNs as therapeutic chemical entities raise several problems, including their susceptibility to enzymatic hydrolysis and poor cellular uptake. Therefore, efficient delivery strategies are required to the introduction of this kind of therapeutic genes into cells. Various transfection agents including viral and nonviral (cationic lipids, polymers, etc), have been utilized to deliver functional proteins and genes into cells, but each system has particular limitations [7, 8].

In recent years, the applications of nanomaterials as gene carriers in biological systems have attracted great interest [9-12]. Gold nanorods (GNRs) are unique class of one-dimensional metal nanostructures and have two 

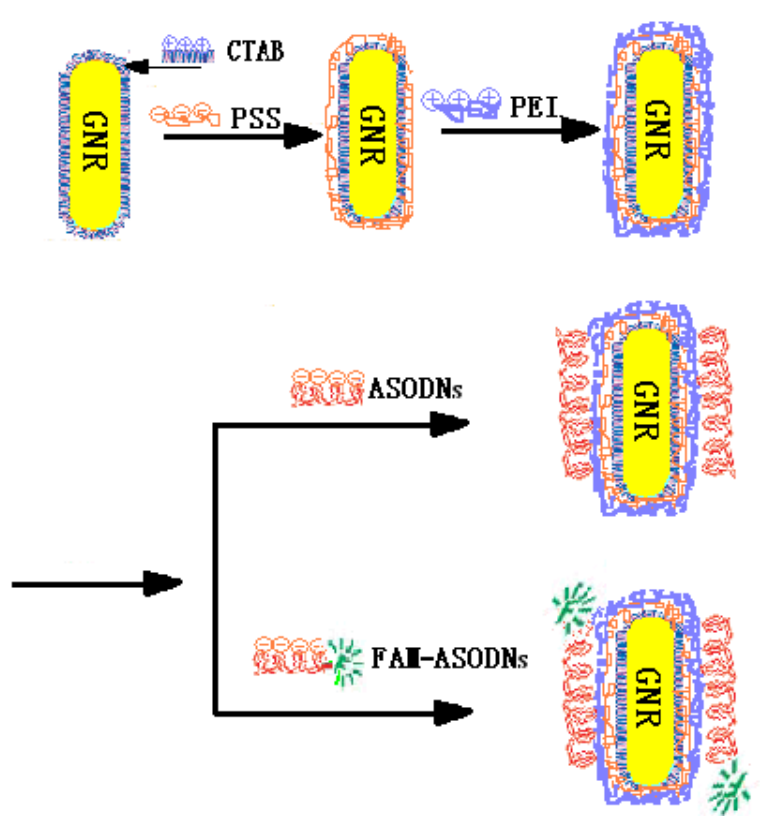

Figure 1. Schematic diagram of the layer-by-layer assembling process of the GNRs-based ASODNs conjugates through electrostatic interactions.

distinct plasmon resonance absorption bands, a longitudinal band corresponding to electron motion along the axis of the particle and the transverse band corresponding to motion along the short axis. The absorption maximum for the longitudinal band shifts itself to longer wavelengths with increasing aspect ratio. In addition, the chemical modification on the gold nanorods surface can be easily achieved. With these tunable optical and structural properties, GNRs show great promise for a range of applications in optical sensing [13, 14], biomedical imaging [15-17], pho-tothermal therapy [18, 19], cancer diagnostic marker [20, 21], biosensing applications [22-24] and gene delivery [25]. Despite these prospects, however, their utilization in the nonviral vectors for ASODNs delivery has been little investigated so far.

Herein, we present the versatile strategy for preparing a novel GNRs-based ASODNs intracellular delivery system through electrostatic layer-by-layer assembling (Figure 1). In this work, in order to reduce cytotoxicity of the GNRs and facilitate further biofunctionalization, the as-prepared GNRs, typically coated with positively charged cetyltrimethylammonium bromide (CTAB), were firstly modified with two successive polyelectrolyte layers of the negatively charged poly (sodium-4-styrene sulfonate) (PSS) and positively charged polyenthylenimine (PEI) via electrostatic interaction and forming PEI/PSS/CTAB/GNRs carrying agent. The polyelectrolytes-coated GNRs were then used as vector for ASODNs. Furthermore, cellular uptake and delivery efficiency of the GNRs-ASODNs conjugates as well as cellular apoptosis induced by the ASODNs transfected with gold nanorods were investigated through confocal microscopy and flow cy- tometry. The results indicate the potential of these metallic nanostructures for use as novel vectors in gene therapeutic applications.

\section{Materials and methods}

\subsection{Materials}

Cetyltrimethylammonium bromide (CTAB), hydrogen tetrachloroaurate(III) trihydrate $\left(\mathrm{HAuCl}_{4} \cdot 3 \mathrm{H}_{2} \mathrm{O}\right.$, $99.9 \%)$, silver nitrate $\left(\mathrm{AgNO}_{3}\right)$, L-ascorbic acid(AA, $99.5 \%)$, and sodium borohydride $\left(\mathrm{NaBH}_{4}, 99 \%\right)$ were purchased from Sinopharm Che-mical Rea-gent Co., Ltd. Poly(sodium-4-styrene-sulfonate) (PSS, Mw 70 000g/mol) and poly-enthylenimine ( PEI, $25 \mathrm{~K}$ ), were obtained from Aldrich. The human telomerase antsense oligodeoxy-nucleotides (ASODNs) sequence used in the current work was 5'-GGAG CGCGCGGCATCGC GGG-3' and modified with fluo-rescently labeled on 5' end (FAM-GGAGCGC GCGGCATCGCGGG-3'), which were obtained from Shanghai Sangon Biological Engineering Technology \& Services Co., Ltd.

\subsection{Synthesis of Gold Nanorods}

Gold Nanorods were synthesized according to the protocols described by Murphy et al [34,35]. Briefly, $0.25 \mathrm{mM} \mathrm{HAuCl}_{4}$ in $0.1 \mathrm{M}$ CTAB solution was reduced by ice-cold $\mathrm{NaBH}_{4}(0.01 \mathrm{M})$ to yield small nanoparticles (less than $5 \mathrm{~nm}$ ) as the $\mathrm{Au}$ seeds solution. $15 \mu \mathrm{L}$ of seed solution was added to a growth solution $(30 \mathrm{ml})$ containing $0.1 \mathrm{M} \mathrm{CTAB}, 0.4 \mathrm{mM} \mathrm{HAuCl} 4,0.06 \mathrm{mM} \mathrm{Ag}$ $\mathrm{NO}_{3}, 0.64 \mathrm{mM} \mathrm{AA}$, and the gold nanorods were then obtained after at least $3 \mathrm{~h}$.

\subsection{Preparation of polyelectrolyte-coated GNRs and GNRs-ASODNs conjugates}


The unpurified gold nanorods (containing excess CTAB) were centrifuged twice at $14000 \mathrm{rpm}$ for 10 min and resuspended in $1 \mathrm{ml} \mathrm{NaCl}(1 \mathrm{mM})$ and PSS $(200 \mu \mathrm{L} 10 \mathrm{mg} / \mathrm{ml}$ ) .PSS is a negatively charged polyelectrolyte which coats the positively charged CTAB bilayer of gold nanorods. Following that, using the same strategy, the negative charged surface of $\mathrm{PSS} / \mathrm{CTAB} / \mathrm{GNRs}$ were reconverted to positive charge by further coating with a layer of PEI polyelectrolyte. Between coating processes, excess polymer was removed by centrifugation. At the completion of the coating process, nanorods were centrifuged twice and resuspended in distilled water to produce a PEI/ PSS/CTAB/GNRs solution.

The positively charged $\mathrm{PEI} / \mathrm{PSS} / \mathrm{CTAB} / \mathrm{GNRs}$ were then mixed with ASODNs $(0.01 \mathrm{mM}$ ) (or fluorescently labeled on 5'-end FAM-ASODNs), and were vortexed for $2 \mathrm{~min}$ and then shaken $3 \mathrm{~h}$ at room temperature, which finally were stored at $4{ }^{\circ} \mathrm{C}$ for future use. The ASODNs linked to the PEI/PSS/CTAB /GNRs by electrostatic interaction to form the GNRsASODNs conjugates.

UV-vis spectroscopy was performed on a Varian model Cary 100 Scan UV-vis spectrophotometer over the range from 400 to $900 \mathrm{~nm}$. Transmission electron microscopy (TEM) measurements were performed on a JEOL-2011 TEM instrument operating at an accelerating voltage of $200 \mathrm{kV}$. The quantity of the GNRs was carried out by inductively coupled plasma (ICP) measurements, performed on a Varian Vista ICP-AES spectrometer.

\subsection{Cytotoxicity Determination of polyelectrolyte- coated GNRs by MTT Assay}

HeLa cells were cultured in RPMI (Roswell Park Memorial Institute) 1640 medium supplemented with $10 \%$ fetal bovine serum (FBS). Actively growing $\mathrm{HeLa}$ cells were seeded at a density of $1 \times 105$ cells/well of a 96-well tissue culture plate and incubated overnight. The cells were treated with CTAB/GNRs, $\mathrm{PEI} / \mathrm{PSS} / \mathrm{CTAB} / \mathrm{GNRs}$ for $48 \mathrm{~h}$ in quadruplets. Control cells were used without GNRs treatment. At the end of each exposure, the toxicity level of GNRs was assessed by 3-(4,5-dimethylazol-2-yl) -2,5-diphenyl -tetrazolium bromide (MTT) assay. The MTT assay helps in cellviability assessment by measuring the enzymatic reduction of yellow tetrazolium MTT to a purple formazan, as measured at $570 \mathrm{~nm}$ using UV-vis mi-croplate reader (Tecan Co.). All experiments were performed 3 times in quadruplets, and the average of all of the experiments has been shown as cell-viability percentage in comparison with the control experiment, while untreated controls were considered as $100 \%$ viable.

\subsection{Transmission Electron Microscopy (TEM) im- aging of cells treated with polyelectrolyte-coated GNRs}

HeLa cells were seeded at a density of $1 \times 106$ cells in a $60 \mathrm{~mm}$ tissue culture dish and grown overnight. The cells were incubation with PEI/PSS/CTAB/GNRs $(100 \mu \mathrm{M})$ for $6 \mathrm{~h}$ and then the cells were washed thoroughly with chilled PBS, centrifuged into a small pellet, and fixed with $2 \%$ glutaraldehyde in PBS $(0.01 \mathrm{M}, \mathrm{pH}$ 7.4 ) for $120 \mathrm{~min}$, and then washed three times with PBS (10 min every time). The cells were postfixed with $1 \%$ osmium tetraoxide in the same buffer for $30 \mathrm{~min}$, then washed three times with PBS, dehydrated through a series of alcohol concentrations $(30 \%, 50 \%, 70 \%$, $90 \%, 100 \%$ ), embedded in Epon, and sliced to a thickness of $70 \mathrm{~nm}$. Images of the slices images were recorded at $100 \mathrm{kV}$ using a Hitachi 600 transmission electron microscope (TEM).

\subsection{Electrochemical Assay of the Electrostatically layer-by-layer Assemb- ling process of polyelectro- lyte-coated GNRs-ASODNs conjugate}

The glassy carbon electrode (GC, $3 \mathrm{~mm}$ in diameter) was polished to a mirror-like surface with 1.0, 0.3, and $0.05 \mu \mathrm{m}$ alumina slurry followed by rinsing thoroughly with doubly distilled water. The electrodes were successively sonicated in 1:1nitric acid, acetone and doubly distilled water, and then allowed to dry. A $10 \mu \mathrm{L}$ of $1 \mathrm{mM}$ CTAB/GNRs solution was dropped on the surface of pretreated GC electrode and dried at room temperature. For preparation of PSS/CTAB/GNRs modified electrode, the CTAB/GNRs modified elec-trode was dipped into $0.1 \%$ PSS solution for $30 \mathrm{~min}$ at room temperature, then washed away the loosely bounded polymers with double distilled water. Similar process was successively applied by electrostatic interaction to prepare PEI/PSS/CTAB/GNRs, ASODN /PEI/PSS/CTAB/GNRs modified GC electrode.

Electrochemical impedance spectroscopy (EIS) measurements were carried out with using CHI660B electrochemistry workstation ( $\mathrm{CH}$ Instruments). The various GNRs modified GC electrode, a saturated calomel electrode (SCE) and a platinum electrode were used as the working electrode, reference and counter electrode, respectively. The impedance measurements were performed in the presence of $10 \mathrm{mM} \mathrm{K} \mathrm{Ke}_{3}$ $(\mathrm{CN})_{6} / \mathrm{K} 4 \mathrm{Fe}(\mathrm{CN})_{6}$ by applying an ac voltage with $5 \mathrm{mV}$ amplitude in a frequency range from $1 \mathrm{~Hz}$ to $100 \mathrm{kHz}$ under open circuit potential condition. The differences in electron-transfer among a variety of modified electrodes were taken as the signal produced by the layerby-layer electrostatic interaction among GNRs, polyeletrolytes, and ASODNs.

\subsection{Cell Transfection assay}

For Confocal microscopy, the Hela cells were fixed on chambered slides and incubated with fluorescence FAM-labeled ASODNs /PEI/PSS /CTAB/GNRs (100 $\mu \mathrm{MPEI} / \mathrm{PSS} / \mathrm{CTAB} / \mathrm{GNRs}, 150 \mathrm{nM}$ ASODNs ) solution for $2 \mathrm{~h}$ at $37^{\circ} \mathrm{C}$ in $5 \% \mathrm{CO}_{2}$ atmosphere. After in- 
cubition, the cells were thoroughly washed with PBS and then observed by a Carl Zeiss LSM 5 PASCAL confocal microscope. An argon laser for FAM excitation at $488 \mathrm{~nm}$ was used for imaging and An oil immersion objective (PlanApo, Magn $=63 \times 1.4$ ) was used for the cellular fluorescence imaging.

For flow cell cytometry, HeLa cells were incubated with FAM-ASODNs (150 nM), FAM-ASODNs/PEI/ $\mathrm{PSS} / \mathrm{CTAB} / \mathrm{GNRs}$ for $2 \mathrm{~h}$ at $37{ }^{\circ} \mathrm{C}$ in $5 \% \mathrm{CO}_{2}$ atmosphere, respectively. After $2 \mathrm{~h}$, the culture was renewed, and the cells were washed with PBS. The cells were then analyzed by flow cytometry. These samples were tested under the same experimental conditions. The delivery efficiency was calculated as the percentage of fluorescent cells out of the total number of cells. The fluorescence was detected from the FAM labeled on ASODNs at $488 \mathrm{~nm}$ excitation.

\subsection{Cellular Apoptosis Assay by flow cytometry}

HeLa cells were seeded at a density of $1.5 \times 104$ cells $/ \mathrm{ml}$ and were grown for $24 \mathrm{~h}$ prior to treatment with ASODNs/ PEI/PSS/CTAB /GNRs. The cells were incubated respectively with naked ASODNs $(150 \mathrm{nM})$,

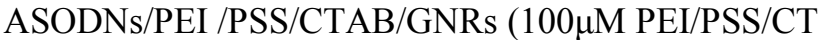
AB/GNRs, $150 \mathrm{nM}$ ASODNs), PEI/PSS/CTAB/GNRs $(100 \mu \mathrm{M})$ and $\mathrm{CTAB} / \mathrm{GNRs}(100 \mu \mathrm{M})$ for $2 \mathrm{~h}$ at $37{ }^{\circ} \mathrm{C}$ in $5 \% \mathrm{CO}_{2}$ atmosphere. After that, the treated cells were thoroughly washed, resuspended in RPMI 1640 me-dium and further incubated for $48 \mathrm{~h}$. The cells were then collected for flow cytometry analysis. We used the PI staining method to identify dead cells, if any, caused by exposure of the cells to ASODNs transfected by PEI/PSS/CTAB/GNRs.

\section{Results and discussion}

The GNRs were prepared by the seed-mediated growth method in $\mathrm{CTAB}$ surfactant solution and via CTAB-directed approach were stabilized by a positively charged bilayer. As it is well known that CTAB coating onto the surface of GNRs is problematic for cytotoxicity and bioconjugation, surface modify-cation of the as-synthesized gold nanorods with biocompatible and functionalization-friendly stabilizing agents has become an important step to realize these functional nanorods for biomedical and biological applications [26-28]. In our experiment, the CTAB-GNRs were further coated with multilayers of polyeletroleytes by an electrostatic layer-by-layer assembling technique in order to mask the CTAB layer and to generate the biocompatible positively charged GNRs as carriers for delivering the antisense oligonucleotides. The UV-vis spectra of the GNRs as a function of different polymer coatings are shown in Figure 2. The spectra showed typical absorption characteristics of GNRs. The longitudinal and tran-sverse plasmon resonance bands are located at $\sim 727 \mathrm{~nm}$ and $521 \mathrm{~nm}$, respectively. It can be seen that there is a small red shift $(\sim 9 \mathrm{~nm})$ in the longitudinal plasmon band maxima after coating with two layers of polymer PSS and PEI (727 nm for CTAB/GNRs and $736 \mathrm{~nm}$ after two layers of polyelectrolyte coating). It is known that the nanocrystal plasmon band is dictated by the local dielectric function. Therefore, the observed red shift is consistent with the changes in the local refractive index from that of water to that of polyelectrolytes [29]. The water dispersible $\mathrm{PEI} / \mathrm{PSS} / \mathrm{CTAB} / \mathrm{GNR}$ were observed to be colloidally stable for several weeks at room temperature.

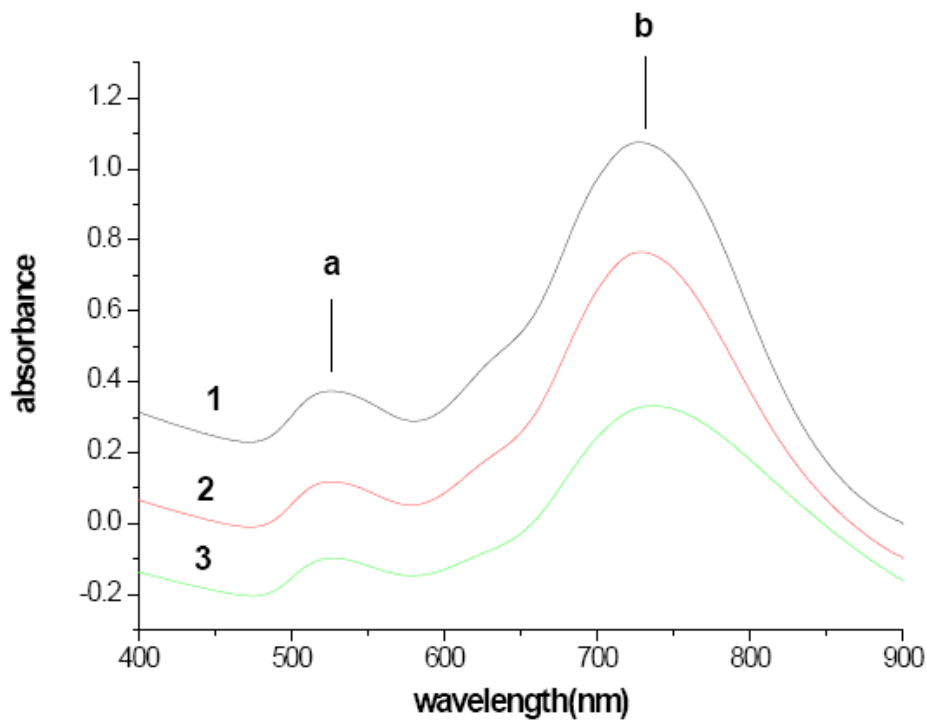

Figure 2. UV-vis spectra of gold nanorods at different stages of surface modification: curve 1, as-prepared CTAB/ GNRs; curve 2, PSS/CTAB/GNRs; curve 3, PEI/PSS/CTAB/GNRs; Features a and $\mathrm{b}$ in the curves correspond to transverse and longitudinal plasmon bands for gold nanorods. 


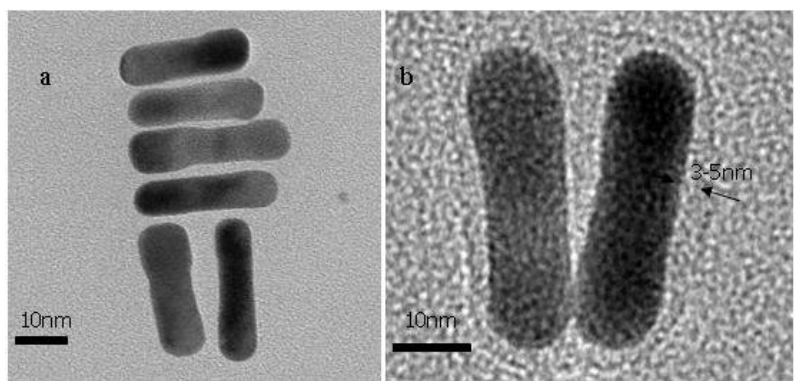

Figure 3. TEM images of (a) as-prepared CTAB/GNRs and (b) PEI/PSS /CTAB/GNRs.

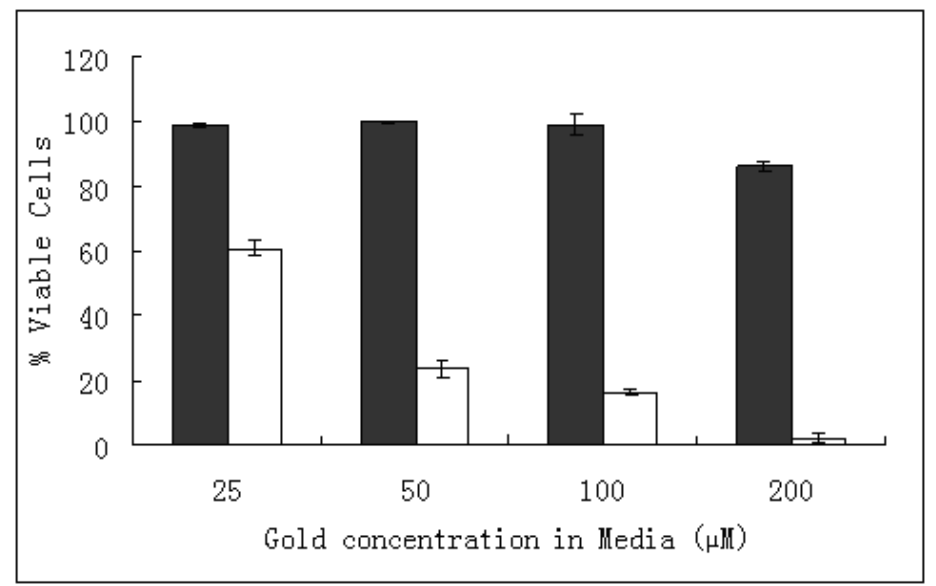

Figure 4. Viabilities of HeLa cells after incubation with PEI/PSS/CTAB/GNRs (closed bars) and CTAB/GNRs (open bars). Data represent mean values for $n=3$.

Direct visualization of polymer coating of the GNRs can be achieved by TEM measurements. TEM images indicated a high yield of GNRs structures with an average length of $30 \mathrm{~nm}$ and average diameter of 10 $\mathrm{nm}$ (aspect ratio of 3). Figure 3 shows the highmagnification TEM micrographs of (a) the uncoated gold nanorods and (b) gold nanorods coated with two layers of PEI and PSS polyelectrolytes. It can be observed that there is a faint layer surrounding the darker rods, indicating that GNRs are uniformly coated by the multilayer polyelectrolytes. The thickness of the polymer coating of GNRs was estimated to be $\sim 3-5 \mathrm{~nm}$.

To examine their biocompatibility of polyelectrolyte-coated GNRs, the cytotoxicity study was conducted to evaluate their effect on Hela cell line by 3(4,5-dimethylazol-2-yl)-2,5-diphenyl-etrazolium bromide (MTT) assay. In the MTT assay, the ab-sorbance of formazan (produced by the cleavage of MTT by dehydrogenases in living cells) at $570 \mathrm{~nm}$ is directly proportional to the number of live cells. It can be clearly seen from Figure 4 that the GNRs display marked cytotoxicity even at low dosages. In compa-rison, for the cells treated with PEI/PSS/CTAB/GNRs, the cell viability was greatly improved and $80 \%$ of the cells even at the maximum dosage $(200 \mu \mathrm{M})$ was observed. The results indicate the modification of poly-electrolyte multilayers onto the surface of GNRs make a dramatic decrease in cytotoxicity. In addition, we also examined whether the polyelectrolyte-coated GNRs were capable of being taken up into the cells using transmission electron microscopy. It can be seen from TEM images of Hela cells after incubation with the polyelectrolytecoated GNRs (Figure 5) that the GNRs have located in the cytoplasm, indicating their effective into cells through possible endocytosis uptaking process. Therefore, based on their biocompatibility and cellular internalizing ability, we used the polyelec-troyte-coated GNRs as a transfection vehicle for intra-cellular delivery of antisense oligonucleotides.

The positively charged polyelectroyte-coated GNRs were then mixed to the negatively charged antisense oligonucleotides to construct the GNRs-ASODNs conjugates through the electrostatic interaction. As electrochemical impedance spectroscopy (EIS) in electrochemistry is considered as an effective approach for monitoring the electrostatic layer-by-layer assembling process $[30,31]$, this technique could be employed to confirm the formation of the GNRs-ASODNs conjugates. Figure 6 shows representative Nyquist diagrams of the electrochemical impedance spectra of 


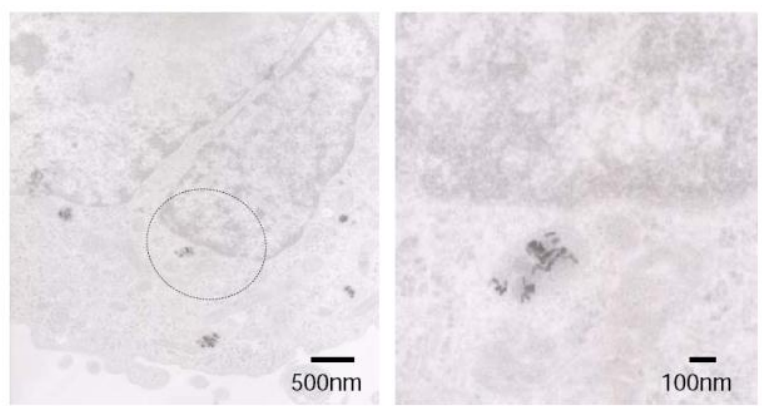

Figure 5. (a) TEM image of HeLa cells with PEI/PSS/CTAB/GNRs. (b) The circled area in part a is shown at higher magnification, showing the PEI/PSS/CTAB/GNRs inside the cell cytoplasm.

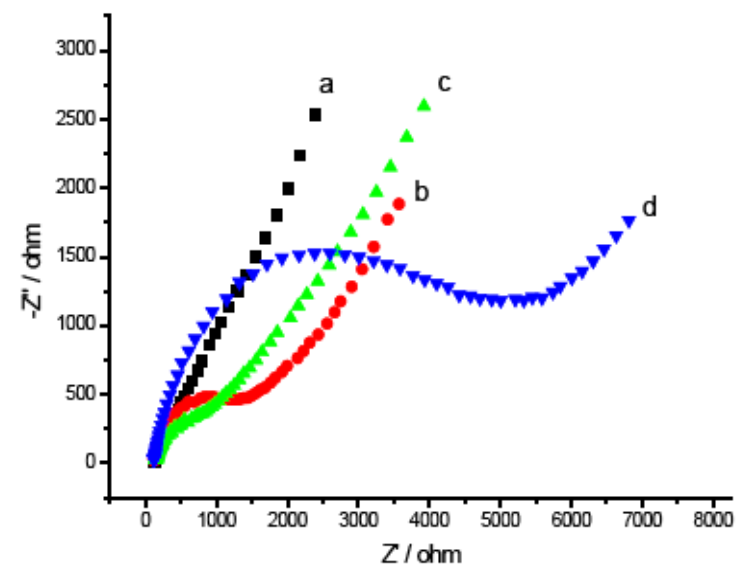

Figure 6. Nyquist plots for electrochemical impedance spectroscopy (EIS) measurements of various modified GC electrode in the presence of $10 \mathrm{mmol} / \mathrm{L}[\mathrm{Fe}(\mathrm{CN}) 6] 3-/ 4$ - with $0.1 \mathrm{~mol} / \mathrm{L} \mathrm{KCl}$ as the supporting electrolyte: (a) CTAB/GNRs, (b) PSS/CTAB/GNRs, (c) PEI/PSS/CTAB/GNRs, (d) ASODNs/ PEI/PSS/CTAB/GNRs.

CTAB/GNRs (curve a), PSS/CTAB /GNRs (curve b), $\mathrm{PEI} / \mathrm{PSS} / \mathrm{CTAB} / \mathrm{GNRs}$ (curve c) and ASO DNs/PEI/PSS/CTAB/GNRs (curve d) modified electrode in the presence of $[\mathrm{Fe}(\mathrm{CN}) 6]^{3 / 4}$ as a redox probe. As shown in Figure 6, each of the impedance spectra includes a semicircle portion and a linear line portion, which correspond to the electron transfer process and diffusion process, respectively. The diameter of the semicircle represents the electron-transfer resistance at the electrode surface. As shown in Figure 6, there is very small semicircle diameter corresponding to very low electron-transfer resistance observed at CTAB/ GNRs modified electrode (curve a). After stepwise coating of polyelectrolytes, the electron-transfer resistances of the PSS/CTAB/GNRs (curve b), PEI/PSS/ CTAB/GNRs(curve c) and ASODNs/PEI/PSS/ CTAB /GNRs (curve d) modified electrode are about 1.5, 0.8, and $5.1 \mathrm{k} \Omega$, respectively, exhibiting this layer-by-layer assem-bling process on the surfaces of the CTAB/GNRs. The EIS results suggest that the PSS, PEI and ASODNs were successively adsorbed to the GNRs by electro-static interaction, which can be explained that the negatively charged PSS polymer coating on the surface of the GNRs relatively restricted the electron transfer of $\mathrm{Fe}(\mathrm{CN})^{3-1 / 4}$ redox couple, successive adsorption of the positively charged PEI polymer facilitated the electron transfer of the redox couple and further adsorption of negatively charged ASODNs relatively hindered the electron transfer of this redox couple. We further investigated the avai-lability on cell interna-lization of the GNRs-ASODNs conjugates. A combi-nation of confocal microscopy and flow cytometry was used to make qualitative and quantitative assay of PEI/PSS/CTAB/GNRs delivery into the HeLa cells. Figure 7 represents confocal ima-ges of HeLa cells incubated with the GNRs-ASODNs conjugates. To visualize intracellular uptake of the ASODNs-loading GNRs conjugates, fluore-scence FAM-labeled ASODNs were used for the complex formation. It can be clearly seen in confocal micro-scopy images that FAM-ASODNs/PEI/PSS/CTAB/G NRs have entered into the cell cyto-plasm and nucleus from the observation of the FAM fluorescent signal (green) within cells. And the conjugates are more concentrated in the nucleus of the HeLa cells. This result suggest that after transported inside the cell cytosol by the PEI/PSS/CTAB/GNRs carriers, 


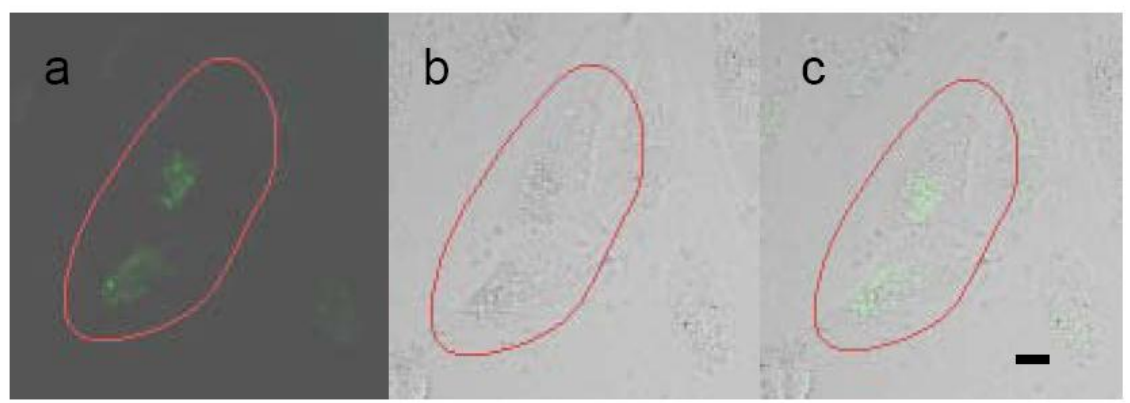

Figure 7. Confocal fluorescence images of HeLa cells incubated with the fluorophore labeled FAM-ASODNs /PEI/PSS/CTAB/GNRs for $2 \mathrm{~h}$. a) intracellular FAM-ASODNs/PEI/PSS/CTAB/GNRs (green fluorescence); b) visible light image; $c$ ) merge image (combined light and fluorescence images). Scale bars $=5 \mu \mathrm{m}$.

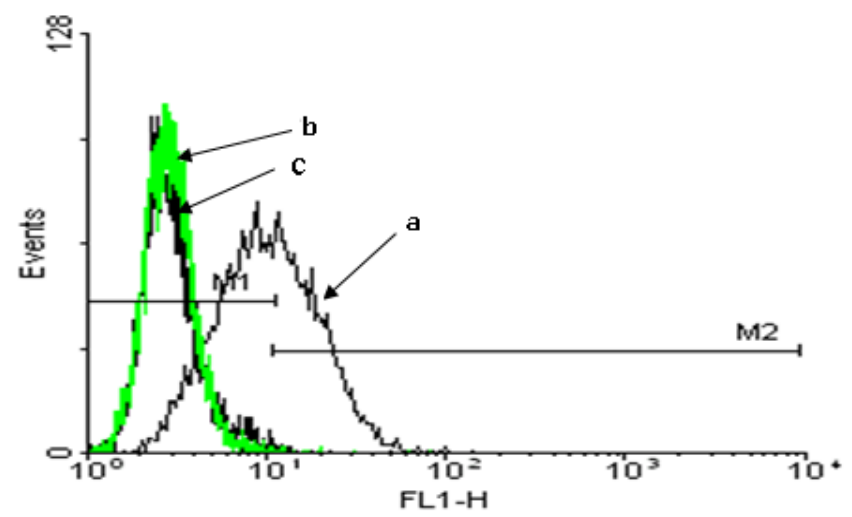

Figure 8. Flow cytometry of cells incubated with FAM-ASODNs/PEI/ PSS/CTAB/GNRs (a) and FAM-ASODNs (b) as compared to unlabeled cells (c) demonstrates the ability to quantify the delivery efficiency of PEI/PSS/CT $\mathrm{AB} /$ GNRs for ASODNs delivery in HeLa cells. After washings, the cells were analyzed by flow cytometry. The fluorescence was detected from the FAM tagged on ASODNs.

ASO-DNs could be delivered into cell nuclei for inhibiting the target mRNA of telomerase, which was possibly ascribed to its special characteristic of strong nucleus localization of ASODNs as reported $[32,33]$.

A flow cytometry enabled the quantitative assay of ASODNs/PEI/PSS/CTAB/GNRs delivery into HeLa cells. HeLa cells incubated with either FAM-ASODNs/ $\mathrm{PEI} / \mathrm{PSS} / \mathrm{CTAB} / \mathrm{GNR}$ or naked FAM-ASODNs for 2 $\mathrm{h}$ were analyzed by using flow cytometry to evaluate the delivery efficiency of PEI/PSS/CTAB/GNRs for ASODNs delivery and using FAM as fluorescence labeling. Figure 8 shows the cellular delivery efficiency of FAM-ASODNs/PEI/PSS/CTAB/GNRs is about $45.5 \%$, in contrast, almost no cellular internalization was observed for FAM-ASODNs alone. This suggested that while ASODNs were unable to traverse across cell membranes by themselves, PEI/PSS/CTAB/GNRs were effective in transporting DNA inside cells and could be utilized as efficient gene delivery vectors, which were attributed to the stable GNRs conjugates prevent-ing ASODNs from enzyme degradation.

Finally, we monitored cellular apoptosis induced by the ASODNs transfected by polyelectrolyte-coated GNRs. Flow cytometry was used to quantitatively evaluate the cell apoptosis efficiency after treating HeLa cells with ASODNs/PEI/PSS/CTAB/GNRs, PEI/ PSS/
CTAB/GNRs and naked ASODNs, respectively, and further incubation for a period of time $(48 \mathrm{~h})$. As shown in Figure 9, we observed significantly higher percentage of apoptosed cells treated with the GNRsASODNs conjugates than those treated with polyelectrolyte coated GNRs or naked ASODNs, indicating the enhanced cellular delivery of ASODNs by PEI/PSS/CT $\mathrm{AB} / \mathrm{GNR}$ can much more effectively inhibit telomerase activity and subsequently induce the cell apoptosis. Therefore, PEI/PSS/CTAB/GNRs could be used as suitable nanocarriers for therapeutic gene delivery applications due to its high surface area, efficient intracellular transporting ability and bio-compatibility.

\section{Conclusions}

In this study, we have prepared a novel GNRsbased ASODNs delivery system via electrostatically layer-by-layer assemblying, in which biocompatible multilayer polyelectrolyte-coated gold nanorods were used as vector and ASODNs as therapeutic gene. The results showed efficient intracellular delivery and improved antitumor activity of ASODNs by PEI/PSS/ CTAB/GNRs carriers. Therefore, these polyelectrolytes coated GNRs could hold great promising for biological delivery applications and gene therapy. 


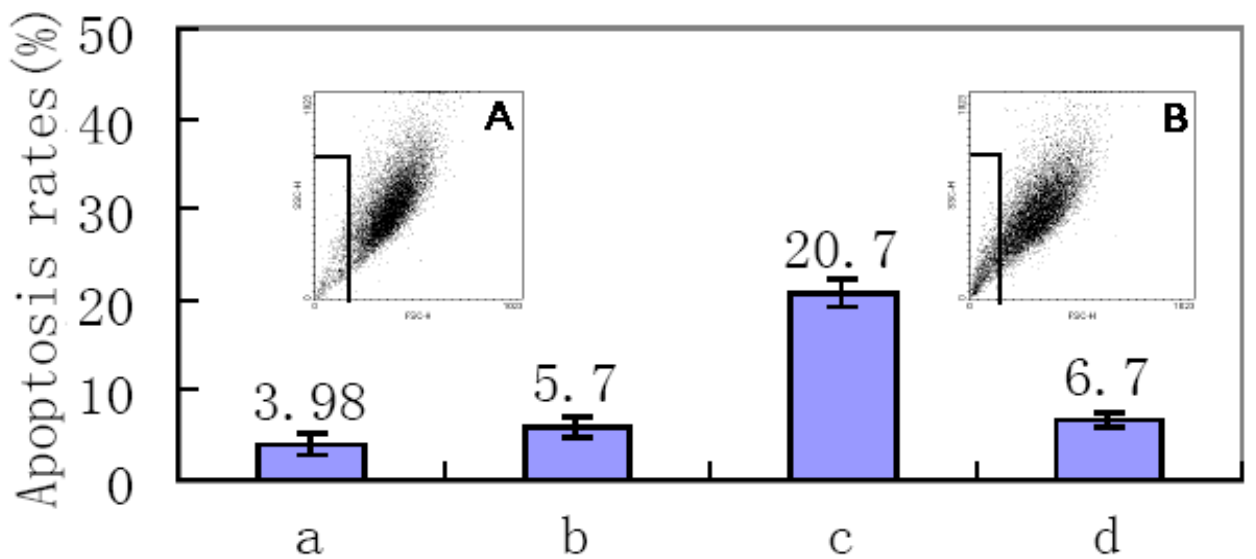

Figure 9. Flow cytometric assay for (a) untreated control HeLa cells and cells treated with (b) PEI/PSS /CTAB/GNRs, (c) ASODNs/PEI/PSS/CTAB/GNRs, and (d) naked ASODNs. The cells were harvested, washed in PBS, fixed in $70 \%$ ethanol, RNase treated, stained with propidium iodide and used for flow cytometric analysis. (Inset: each black point corresponds to a single cell. The right quadrant corresponds to living cells, the left quadrant corresponds to apoptotic cells. (A) cells treated with PEI/PSS/CTAB/GNRs (B) cells treated with ASODNs /PEI/PSS/CTAB/GNRs ).

\section{Acknowledgements}

This work was supported by LADP-SHNU (DZL806), NSFC (20773088), National Basic Research Program of China (2008CB617504), Program for New Century Excellent Talents in University (NCET-08-0897), Shanghai Sci. \& Tech. and Education Committee (08QH14020, 09SG43, 09zz137 and S30406).

\section{References}

1. Biroccio1 A, Leonettil C, Zupi G. The future of antisense therapy: combination with anticancer treatments. Oncogene 2003; 22: 6579-6588. doi:10.1038/sj.onc. 1206812

2. Stahel RA, Zangemeister-Wittke U. Antisense oligonucleotides for cancer therapy an overview. Lung Cancer 2003; 41: 581-588. doi:10.1016/S0169-5002(03) 00147-8

3. Greider CW, Blackburn EH. Identification of a specific telomere terminal transferase activity in Tetrahymena extracts. Cell 1985; 43: 405-413. doi:10.1016/0092$\underline{\text { 8674(85)90170-9 }}$

4. R. Hodes. Molecular targeting of cancer: Telomeres as targets. Proc Natl Acad Sci USA 2001; 98: 7649-7651. doi:10.1073/pnas.151267698

5. Feng J, Funk WD, Wang SS, Weinrich SL, Avilion AA, Chiu CP, Adams RR, Chang E, Allsopp RC, Yu J. The RNA component of human telomerase. Science 1995; 269: 1236-1241. doi:10.1126/science.7544491

6. White LK, Wright WE, Shay JW. Telomerase inhibitors. Trends in biotechnology 2001; 19: 114-120. doi:10.1016/S0167-7799(00)01541-9

7. Sokolova V, Epple M. Inorganic nanoparticles as carriers of nucleic acids into cells. Angew Chem Int Ed 2008; 47: 1382-1395. doi:10.1002/anie.200703039

8. Mintzer MA, Simanek EE. Nonviral vectors for gene deliv- ery. Chem Rev 2009;109: 259-302. doi:10.1021/cr80 $\underline{0409 \mathrm{e}}$

9. Liu Z, Winters M, Holodniy M, Dai H. siRNA delivery into human $\mathrm{T}$ cells and primary cells with carbon nanotube transporters. Angew Chem Int Ed 2007: 46, 2023-2027. doi:10.1002/anie.200604295

10. Jia NQ, Lian Q, Shen HB, Wang C, Li XY, Yang ZN. Intracellular delivery of quantum dots tagged antisense oligodeoxynucleotides by functionalized multiwalled carbon nanotubes. Nano Lett 2007; 7: 2976-2980. doi: $10.1021 / \mathrm{nl} 071114 \mathrm{c}$

11. Rosi NL, Mirkin CA. Nanostructures in biodiagnostics. Chem.Rev. 2005; 105: 1547-1562. doi:10.1021/cr030067f

12. Rosi NL, Giljohann DA, Thaxton CS, Lytton-Jean AKR, Han MS, Mirkin CA. Oligonucleotide-modified gold nanoparticles for intracellular gene regulation. Science 2006; 312:1027-1030. doi:10.1126/science.1125559

13. Alekseeva AV, Bogatyrev V A, Dykman LA, Khlebtsov BN, Trachuk LA. Preparation and optical scattering characterization of gold nanorods and their application to a dotimmunogold assay. Appl Opt 2005; 44: 6285-6295. doi:10.1364/AO.44.006285

14. Yu C, Irudayaraj J. Multiplex biosensor using gold nanorods. Anal Chem 2007; 79: 572-579. doi:10.1021 lac061730d

15. Huang X, El-Sayed IH, Qian W, El-Sayed M A. Cancer cell imaging and photothermal therapy in the near-infrared region by using gold nanorods. J Am Chem Soc 2006;128: 2115-2120. doi:10.1021/ja057254a

16. Wang H, Huff TB, Zweifel DA, He W, Wei A, Cheng JX. In vitro and in vivo two-photon luminescence imaging of single gold nanorods. Proc Natl Acad Sci USA 2005; 102: 15752-15756.doi:10.1073/pnas.0504892102

17. Tong L, Wei QS, Wei A, Cheng JX. Gold nanorods as contrast agents for biological imaging: optical properties, sur- 
face eonjugation and photothermal effects. Photochemistry and Photobiology 2009; 85: 21-32. doi:10.1111/j.17511097.2008.00507.x

18. Takahashi H, Niidome T, Nariai A, Niidome Y, Yamada S. Gold nanorod-sensitized cell death: microscopic observation of single living cells irradiated by pulsed near-infrared laser light in the presence of gold nanorods. Chem Lett 2006, 35: 500-501. doi:10.1246/cl.2006.500

19. Huff TB, Tong L, Zhao Y, Hansen MN, Cheng JX, Wei A. Hyperthermic effects of gold nanorods on tumor cells. $\mathrm{Na}$ nomedicine $2007 ; \quad 2: \quad 125-132$. doi:10.2217/1743588 $\underline{9.2 .1 .125}$

20. Huang XH, El-Sayed IH, Qian W, El-Sayed MA. Cancer cells assemble and align gold nanorods conjugated to antibodies to produce highly enhanced, sharp, and polarized surface Raman spectra: A potential cancer diagnostic marker. Nano Lett 2007; 7: 1591-1597. doi:10.1021/n10 $\underline{70472 \mathrm{c}}$

21. Yu CX, Nakshatri H, Irudayaraj J. Identity profiling of cell surface markers by multiplex gold nanorod probes. Nano Lett 2007; 7: 2300-2306. doi:10.1021/n1070894m

22. Yang DP, Cui DX. Advances and prospects of gold nanorods. Chem Asian $J$ 2008; 3: 2010-2022. doi:10.1002/asia.200800195

23. Huang X, El-Sayed I. H, W. Qian, El-Sayed MA. Cancer cell imaging and photothermal therapy in the near-infrared region by using gold nanorods. J Am Chem Soc 2006; 128: 2115-2120. doi:10.1021/ja057254a

24. Lee KS, El-Sayed MA. Gold and silver nanoparticles in sensing and imaging: Sensitivity of plasmon response to size, shape, and metal composition. J Phys Chem B 2006; 110: 19220-19225. doi:10.1021/jp062536y

25. Chen CC, Lin YP, Wang CW. DNA-gold nanorod conjugates for remote control of localized gene expression by near Infrared Irradiation. $J$ Am Chem Soc 2006; 128: 37093715. doi: $10.1021 / \mathrm{ja} 0570180$

26. Liao HW, Hafner JH. Gold nanorod bioconjugates. Chem Mater 2005; 17: 4636-4641 doi:10.1021/cm050935k

27. Yu CX, Varghese L, Irudayaraj J. Surface modification of CTAB capped gold nanorods to make molecular probes. Langmuir 2007; 23: 9114-9119. doi:10.1021/la701111e

28. Alkilany AM, Nagaria PK, Hexel CR, Shaw TJ, Murphy CJ, Wyatt MD. Cellular uptake and cytotoxicity of gold nanorods: molecular origin of cytotoxicity and surface effects. Small 2009; 5: 701-708. doi:10.1002/smll.20080 1546

29. Ding H, Yong KT, Roy I. Gold nanorods coated with multilayer polyelectrolyte as contrast agents for multimodal imaging. J Phys Chem C 2007; 111: 12552-12557._doi:10. $\underline{1021 / \mathrm{jp} 0733419}$

30. Katz E, Willner I. Probing biomolecular interactions at conductive and semiconductive surfaces by impedance spectroscopy: routes to impedimetric immunosensors, DNA-sensors, and enzyme biosensors. Electroanalysis 20 03; 15: 913-947. doi:10.1002/elan.200390114

31. Pei R, Cui X, Yang X,Wang E. Assembly of alternating polycation and DNA multilayer films by electrostatic layerby-layer adsorption. Biomacromolecules 2001; 2: 463-468. doi:10.1021/bm0001289
32. Chirila TV, Rakoczy PE, Garrett KL, Lou X, Constable IJ. The use of synthetic polymers for delivery of therapeutic antisense oligodeoxynucleotides. Biomaterials 2002; 23: 321-342. doi:10.1016/S0142-9612(01)00125-9

33. Li SD, Huang L. Targeted delivery of antisense oligodeoxynucleotide and small interference RNA into lung cancer cells. Mol Pharmaceutics 2006; 3: 579-588. doi: $10.1021 / \mathrm{mp} 060039 \mathrm{w}$

34. Sau TK, Murphy CJ. Seeded high yield synthesis of short $\mathrm{Au}$ nanorods in aqueous solution. Langmuir 2004; 20: 6414-6420. doi:10.1021/la049463z

35. Murphy CJ, Sau TK, Gole AM, Orendorff CJ, Gao J, Gou L, Hunyadi SE, Li T. Anisotropic metal nanoparticles: synthesis, assembly, and optical applications. J Phys Chem B 2005; 109: 13857-13870. doi:10.1021/jp0516846

Received 10 January, 2010; accepted 21 February, 2010; published online 5 March, 2010.

Copyright: (c) $2010 \mathrm{~S}$. Chen et al. This is an open-access article distributed under the terms of the Creative Commons Attribution License, which permits unrestricted use, distribution, and reproduction in any medium, provided the original author and source are credited. 